\title{
A Word from the Editors
}

It is our great pleasure to present to you the 2019 edition of the TESL Canada Journal. In this issue, you will find five articles that each draw attention to different dimensions of language teaching. In the first article, Literacy Engagement in Multilingual and Multicultural Learning Spaces, Theodora Kapoyannis challenges monolingual and monocultural norms in literacy practices with young learners. In this study, the author describes a collaborative project in which young learners drew on culturally relevant texts to bolster their literacy development. The project proved to be valuable in developing vocabulary acquisition through the creation of identity texts that allowed students to use language in ways that were personally meaningful.

The second article draws attention to the professional lives of adult English language teachers. In this article, The Precarious Work of English Language Teaching in Canada, Sherry Breshears examines the nature and conditions of employment of English as a second language (ESL) teachers. By drawing on data that document the experiences of teachers, the author brings to the fore the ways in which multiple factors contribute to precarious employment. She calls for collective mobilization and action on the part of professional associations to address these issues.

In the next article, Teaching in Linguistically and Culturally Diverse Classrooms in Canada: Self-Efficacy Perceptions of Internationally Educated Teachers, the discussion shifts to a focus on Internationally Educated Teachers (IETs) and their self-efficacy beliefs for teaching in linguistically and culturally diverse K-12 classrooms. In this study, researchers Mithila Vidwans and Farahnaz Faez found that IETs reported greater self-efficacy, than non-IETs, in providing culturally responsive pedagogy in multilingual classrooms.

Also situated in the K-12 context, Ana Vintan and Tiffany L. Gallagher explore how ESL teachers and elementary classroom teachers collaborated to support English language learners. In this article, Collaboration to Support ESL Education: Complexities of the Integrated Model, the authors examine multiple sources of data to understand how teachers draw on pedagogy and materials to work together and support learners. The findings highlight specific barriers that proved challenging to ESL teachers and suggest how these efforts can be better supported.

In the final article, The Knowledge Base of L2 Pronunciation Teaching: The Case of a Nonnative-Speaking Teacher, Joshua Gordon draws our attention to a specific pedagogical context - teaching second language pronunciation-and asks how nonnative-speaking teachers of English draw on their professional knowledge to make decisions about pedagogy. Through this case study, Gordon provides insight into the complexity of teacher professional knowl- 
edge, and in particular the ways in which teacher decision-making is influenced by a range of factors, including their experiences as learners.

In addition to these articles, Deniz Toker reviews the series World English 1 (2nd Ed.) and describes how this teaching material, part of the World Englishes series, draws on engaging content from National Geographic and TED to address a range of English language skills. In a second book review, Brock Wojtalewicz provides a comprehensive review of the book Focus on Vocabulary Learning by Marlise Horst. Wojtalewicz describes how specific features of the book provide research-informed strategies that teachers can apply in their classrooms.

This past year, we were also very pleased to receive a number of exciting special issue proposals and selected two special issues for 2019. Earlier this year, we released the special issue, Language and Higher Education, guest edited by Saskia Van Viegen, Meike Wernicke, and Sandra Zappa-Hollman, and we look forward to our next issue Building Capacity for 21st Century Digital ELT Practices, guest edited by Geoff Lawrence, available on-line soon.

In closing, we would like to extend our sincere appreciation to our readers, reviewers, and board members, for your continuing support of the TESL Canada Journal.

Farahnaz Faez (Western University) Antonella Valeo (York University)

\section{Mot des rédactrices}

Nous sommes heureuses de vous présenter l'édition 2019 de la Revue TESL $d u$ Canada. Dans ce numéro, vous trouverez cinq articles qui attireront votre attention sur certaines dimensions de l'enseignement des langues. Dans le premier, intitulé Literacy Engagement in Multilingual and Multicultural Learning Spaces, Theodora Kapoyannis remet en question les normes monolingues et monoculturelles des pratiques d'alphabétisation des jeunes apprenantes et apprenants. Dans cette étude, l'autrice décrit un projet collaboratif au cours duquel de jeunes apprenantes et apprenants ont eu recours à des textes culturellement significatifs pour stimuler le développement de leur littératie. Le projet s'est avéré utile pour le développement de l'acquisition de vocabulaire grâce à la création de textes identitaires qui donnaient à l'étude de la langue une résonnance plus personnelle pour les étudiantes et étudiants.

Le deuxième article jette un regard sur la vie professionnelle dans le cadre de l'enseignement de l'anglais aux adultes. Dans ce texte, intitulé The Precarious Work of English Language Teaching in Canada, Sherry Breshears examine le domaine et les conditions de travail des enseignantes et enseignants d'anglais langue seconde (ESL). En s'appuyant sur des données qui en documentant 
l'expérience, l'autrice fait ressortir les multiples facteurs qui contribuent à la précarité de l'emploi. Elle encourage les associations professionnelles à se mobiliser et à agir pour remédier à ces problèmes.

Dans l'article suivant, intitulé Teaching in Linguistically and Culturally Diverse Classrooms in Canada: Self-Efficacy Perceptions of Internationally Educated Teachers, la discussion porte sur les enseignantes et enseignants formés à l'étranger (IETs) et évalue la mesure dans laquelle ils se sentent personnellement à même d'offrir un enseignement efficace dans des salles de classe de la maternelle à la $12^{\mathrm{e}}$ année caractérisées par une diversité linguistique et culturelle. Dans cette étude, les chercheuses Mithila Vidwans et Farahnaz Faez concluent que les enseignantes et enseignants formés à l'étranger se sentent mieux préparés pour offrir une pédagogie adaptée aux réalités culturelles dans une salle de classe multilingue que ne le font les enseignantes et enseignants formés ailleurs qu'à l'étranger (non-IETs).

Se situant elles aussi dans le contexte de la maternelle à la $12^{\mathrm{e}}$ année, Ana Vintan et Tiffany L. Gallagher explorent la façon dont des enseignantes et enseignants d'anglais langue seconde (ESL) et des enseignantes et enseignants au primaire ont collaboré au soutien de l'enseignement aux apprenantes et apprenants de la langue anglaise (ELLs). Dans cet article, intitulé Collaboration to Support ESL Education: Complexities of the Integrated Model, les autrices examinent des sources de données multiples afin de comprendre comment les enseignantes et enseignants s'appuient sur la pédagogie et les ressources didactiques pour travailler ensemble et soutenir les apprenantes et apprenants. Les constatations mettent en lumière les obstacles particuliers auxquels sont confrontés les enseignantes et enseignants d'anglais langue seconde et proposent des façons de mieux appuyer leurs efforts.

Dans le dernier article, intitulé The Knowledge Base of L2 Pronunciation Teaching: The Case of a Nonnative-Speaking Teacher, Joshua Gordon attire notre attention sur un contexte pédagogique particulier-l'enseignement de la prononciation d'une langue seconde-et se pose la question de savoir comment les professeurs d'anglais non natifs puisent dans leurs connaissances professionnelles pour prendre des décisions d'ordre pédagogique. Par le biais de cette étude de cas, Gordon lève le voile sur la complexité des compétences professionnelles d'une enseignante particulière, notamment en ce qui concerne la façon dont la prise de ses décisions peut être influencée par une gamme de facteurs comme, entre autres, ses propres expériences d'apprentissage.

En plus de ces articles, Deniz Toker signe un compte rendu de la série World English 1 (2nd Ed.) et décrit comment cette ressource pédagogique qui s'inscrit dans la série World Englishes utilise des contenus tirés du magazine National Geographic et de TED pour l'enseignement d'une gamme de compétences en anglais. Dans un second compte rendu, Brock Wojtalewicz présente une recension exhaustive de Focus on Vocabulary Learning, un livre de Marlise Horst, en décrivant comment certaines caractéristiques spécifiques de 
cet ouvrage fournissent des stratégies qui sont éclairées par des recherches et auxquelles les enseignantes et enseignants peuvent recourir en classe.

Au cours de la dernière année, nous avons eu le grand plaisir de recevoir de nombreuses suggestions stimulantes pour de futurs numéros spéciaux de notre revue, et nous en avons sélectionné deux pour 2019. Plus tôt cette année, nous avons lancé Language and Higher Education, un numéro spécial sur la langue et l'enseignement supérieur préparé par les rédactrices invitées Saskia Van Viegen, Meike Wernicke et Sandra Zappa-Hollman, et nous sommes à préparer notre prochain numéro, Building Capacity for 21st Century Digital ELT Practices, qui portera sur les pratiques dans le domaine des Cours de langue de niveau avancé (CLNA) et sera placé sous la direction du rédacteur invité Geoff Lawrence et bientôt disponible en ligne.

En terminant, nous aimerions présenter nos sincères remerciements à nos lectrices et lecteurs, à nos réviseuses et réviseurs et aux membres de notre conseil d'administration pour le soutien qu'elles et ils continuent d'offrir à la Revue TESL du Canada.

Farahnaz Faez (Université Western) Antonella Valeo (Université York) 